\title{
Augmentation of autologous hamstring graft during anterior cruciate ligament reconstruction using the bone chip technique
}

\author{
Kyung Wook Nha
}

Received: 14 May 2010/Accepted: 28 July 2010/Published online: 14 September 2010

(C) Springer-Verlag 2010

Dear Editors,

We appreciate the comments made by Milankov et al. with regards to our paper "Augmentation of autologous hamstring graft during anterior cruciate ligament reconstruction using the bone chip technique" [2]. We have read a letter written by Milankov about our manuscript, and reviewed the techniques published by Milankov et al. [1] and Takeuchi et al. [3]. In respect that augment the bone at the end of graft material, that technique was similar to ours. Especially, as Milankov et al. suggested, the harvested bony material from tibial tunnel with the cancellous bone extractor is thought to be better because of no additional bone damage.

Although we possess the coring reamer (Arthrex, Naples, FL, USA), we do not use that device. It requires additional procedure and high cost to gain bony material compared with our technique.

We make the femoral tunnel through the tibial tunnel (not AM portal), and we perform the notchplasty as routine procedure to obtain a better visualization, and to prevent the graft impingement. That procedure is also for getting the bone chip. If we need to augment with a thin graft, we simply obtain the bone with the notchplasty osteotome (Arthrex, Naples, FL) (Fig. 1).

As Takeuchi mentioned, obtaining the bone block from the medial aspect of the tibial tuberosity would need to cut into 2 pieces, and the cancellous bone column harvested from same site also need to cut into 2 blocks sagittally using core reamer. After graft fixation, half of the cancellous bone

K. W. Nha $(\bowtie)$

Department of Orthopedic Surgery,

Ilsanpaik Hospital, Inje University, Ilsan, Korea

e-mail: kwnhamj@hotmail.com
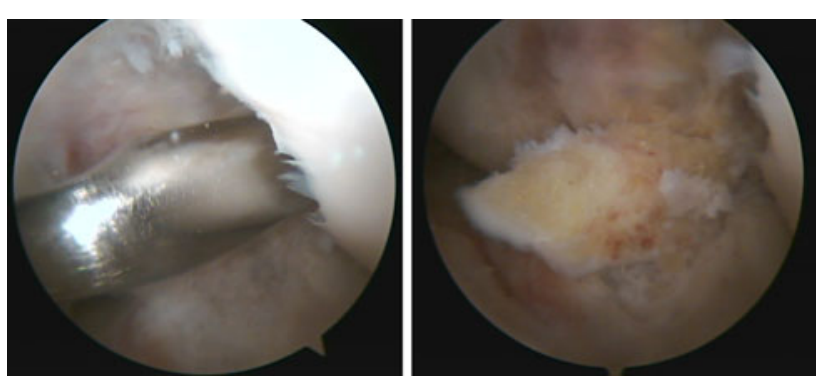

Fig. 1 Arthroscopic view showing obtained bone with the notchplasty osteotome (Arthrex, Naples, FL)

column harvested from the tibial bone tunnel should be implanted at the site of the bone groove on the medial side of the tibial tuberosity. Compared with ours, that technique also requires core reamer. It is a more complicated procedure with high technical demand and a longer operation time. It is generally believed that tibial fixation site can be weakest in early phase after graft fixation. Unfortunately, the harvest site is located near the tibial fixation site around the entrance of the tibial tunnel in Takeuchi method.

\section{References}

1. Milankov M, Miljkovic N, Savic D (2007) Anterior cruciate ligament reconstruction using compressed bone-hamstring-bone graft. Arthroscopy 23:442.e1-442.e3

2. Nha KW, Shetty GM, Ahn JH, Lee YS, Chae DJ, Nam HW, Lee DH (2010) Augmentation of autologous hamstring graft during anterior cruciate ligament reconstruction using the bone chip technique. Knee Surg Sports Traumatol Arthrosc 18:89-91

3. Takeuchi R, Saito T, Mituhashi S, Suzuki E, Yamada I, Koshino T (2002) Double-bundle anatomic anterior cruciate ligament reconstruction using bone-hamstring-bone composite graft. Arthroscopy 18:550-555 